\title{
Effect and safety of tenecteplase in stroke patients with atrial fibrillation. Post-hoc analysis of the Norwegian Tenecteplase Stroke Trial NOR-TEST
}

\begin{abstract}
Anne Hege Aamodt, ' Nicola Logallo, ${ }^{2}$ Lars H.Alteheld,' Martin Kurz, ${ }^{3}$ Annette Fromm, ${ }^{2}$ Lars Thomassen, ${ }^{2}$ Halvor Næss ${ }^{2}$
'Department of Neurology, Oslo University Hospital, Norway, ${ }^{2}$ Department of Neurology, Haukeland University Hospital, Norway ${ }^{3}$ Department of Neurology, Stavanger University Hospital, Norway
\end{abstract}

\section{Background}

Although there has been a revolutionary progress of endovascular treatment, intravenous thrombolysis remains the pillar of reperfusion therapy. Patients with atrial fibrillation (AF) are at increased risk of stroke and these strokes tend to be the most serious ones. It is therefore essential to find the most effective thrombolytic treatment in order to resolve also cardiac emboli more sufficiently. Alteplase (tPA) has well recognized limitations, and there is an imperative to continue efforts to find more efficient and safer lytic treatment in acute ischemic stroke.

NOR-TEST was the first randomized controlled phase 3 trial to investigate the safety and efficacy of tenecteplase versus a tPA in acute ischemic stroke.

\section{Aim}

The purpose of this post hoc analysis of the NOR-TEST study was to assess the effect and safety of tenecteplase versus tPA in patients with acute ischemic stroke and AF, and to assess the outcome in stroke patients with AF compared to patients with sinus rhythm.

\section{Material and methods}

The patients were randomized to receive either tenecteplase $0.4 \mathrm{mg} / \mathrm{kg}$ or alteplase $0.9 \mathrm{mg} / \mathrm{kg}$. Atrial fibrillation was defined as documented paroxysmal or chronic prior to the ischemic stroke or documented atrial fibrillation during the hospital stay. Patients with atrial fibrillation were compared to patients without atrial fibrillation.

Table 1. Baseline characteristics and outcome in patients with $\mathrm{AF}$ versus sinus rhythm (SR).

\begin{tabular}{l}
\hline Variables \\
\hline Age \\
\hline Female \\
\hline NIHSS score on baseline \\
\hline Prior cerebral infarction \\
\hline Active smoking \\
\hline NIHSS score at 24 hours \\
\hline mRS at day 7 \\
\hline mRS at 3 months \\
\hline Any intracerebral \\
\hline hemorrhage
\end{tabular}

\begin{tabular}{|c|c|c|c|c|c|}
\hline \multicolumn{2}{|c|}{ All patients } & & \multicolumn{2}{|c|}{ Per protocol } & \\
\hline $\mathrm{N}=183$ & $\mathrm{~N}=917$ & & $\mathrm{~N}=172$ & $\mathrm{~N}=738$ & \\
\hline $77.0(8.8)$ & $69.8(14.3)$ & $<.001$ & $77.2(8.6)$ & $71.7(12.5)$ & $<.001$ \\
\hline $75(17)$ & $365(83)$ & .77 & $72(21)$ & $275(79)$ & .26 \\
\hline $7.4(6.7)$ & $5.3(4.9)$ & $<.001$ & $7.6(6.8)$ & $5.6(5.2)$ & $<.001$ \\
\hline $38(21)$ & $134(15)$ & .04 & $37(22)$ & $114(15)$ & .054 \\
\hline $24(13)$ & $209(23)$ & .008 & $23(13)$ & $168(23)$ & .006 \\
\hline $5.1(7.6)$ & $3.4(5.6)$ & .001 & $5.4(7.7)$ & $3.8(6.0)$ & .004 \\
\hline $2.1(1.8)$ & $1.5(1.6)$ & $<.001$ & $2.2(1.8)$ & $1.7(1.7)$ & .004 \\
\hline $2.0(1.9)$ & $1.4(1.6)$ & $<.001$ & $2.0(1.9)$ & $1.6(1.7)$ & .003 \\
\hline $28(15)$ & $69(8)$ & .001 & $28(16)$ & $66(9)$ & .004 \\
\hline
\end{tabular}

\section{Results}

Totally 183 patients ( $16.6 \%)$ in the NOR-TEST population $(n=1100)$ were diagnosed with AF. In 120 patients (10.9\%), AF was known prior to the ischemic stroke ( 87 with paroxysmal $A F$ and 33 chronic $\mathrm{AF}$ ) and 63 patients were diagnosed with AF during the acute phase. There was a significantly lower proportion of stroke mimics among patients with AF compared to patients with SR.

In AF patients, hypertension was more frequent in the tenecteplase group and diabetes mellitus more frequent in the tPA group. There were no statistical significant differences in any other baseline characteristics between patients treated with tenecteplase and tPA. Male sex, lower age and NIHSS score were associated with better outcome (per protocol).

Table 2. Baseline characteristics in patients with AF treated with tenecteplase (TNK) versus alteplase.

\begin{tabular}{|l|c|c|c|c|c|c|}
\hline Variables & \multicolumn{3}{|c|}{ All patients } & \multicolumn{3}{c|}{ Per protocol } \\
\hline & TNK & tPA & P & TNK & tPA & P \\
\hline NIHSS on admission & $7.6(7.7)$ & $7.2(5.9)$ & .64 & $7.9(7.9)$ & $7.4(5.9)$ & .64 \\
\hline NIHSS at 24 hours & $6.0(8.6)$ & $4.4(6.7)$ & .18 & $6.4(8.7)$ & $4.7(6.8)$ & .16 \\
\hline NIHSS at 7 days & $3.3(5.7)$ & $3.2(5.5)$ & .87 & $3.6(5.9)$ & $3.4(5.6)$ & .84 \\
\hline mRS $\leq 2$ at day 7 & $39(48)$ & $57(56)$ & .30 & $39(48)$ & $57(56)$ & .30 \\
\hline Mortality at day 7 & $5(6)$ & $1(1)$ & .05 & $5(7)$ & $1(1)$ & .05 \\
\hline mRS $\leq 2$ at 3 months & $50(62)$ & $72(71)$ & .21 & $46(61)$ & $68(71)$ & .16 \\
\hline Mortality at 3 months & $10(12)$ & $7(7)$ & .20 & $10(13)$ & $7(7)$ & .20 \\
\hline Any intracerebral hemorrhage & $12(15)$ & $16(16)$ & .87 & $12(16)$ & $16(17)$ & .88 \\
\hline Symptomatic intracerebral & $3(4)$ & $5(5)$ & .69 & $3(4)$ & $5(5)$ & .70 \\
\hline hemorrhage & & & & & & \\
\hline
\end{tabular}

Table 3. Logistic regression $m R S \leq 2$ at 3 months per protocol.

\begin{tabular}{|l|c|c|c|}
\hline Variables & Odds ratio & $95 \% \mathrm{Cl}$ & $\mathbf{P}$ \\
\hline Male & 2.8 & $1.3-6.1$ & .008 \\
\hline Age & 0.92 & $0.87-0.97$ & .002 \\
\hline NIHSS score on admittance & 0.84 & $0.78-0.90$ & $<.001$ \\
\hline Alteplase versus & 1.8 & $0.8-3.8$ & .15 \\
\hline tenecteplase & & & \\
\hline
\end{tabular}

\section{Conclusions}

This is the first randomized controlled study to report the effect and safety of tenecteplase in acute ischemic stroke in relation to AF. There were no major differences in outcome between the tenecteplase and alteplase group in the subgroup of patients with AF although female sex was associated with more serious strokes and less effect of tenecteplase. 